\title{
Imaging in polymyalgia rheumatica
}

\author{
N. Possemato, C. Salvarani, N. Pipitone \\ Rheumatology Unit, Department of Internal Medicine, Azienda Ospedaliera ASMN, \\ Istituto di Ricovero e Cura a Carattere Scientifico, Reggio Emilia, Italy
}

\begin{abstract}
Polymyalgia rheumatica (PMR) is a chronic, inflammatory disorder of unknown cause clinically characterized by pain and prolonged morning stiffness affecting the shoulders and often the pelvic girdle and neck. Imaging has substantially contributed to defining PMR as a disease mainly involving extra-articular structures. This review article analyses the role of the different imaging techniques in the diagnosis and follow-up of patients with PMR with particular emphasis on the role of ultrasound, PET/CT and MRI.
\end{abstract}

Key words: Rheumatology; Polymyalgia rheumatica; Imaging; Ultrasound; MRI; PET-CT.

\section{INTRODUCTION}

Dolymyalgia rheumatica (PMR) is a chronic, inflammatory disorder of unknown cause. It is almost never seen in people aged 50 years or younger. Clinically, the disorder is characterized by pain and prolonged morning stiffness affecting the shoulders and often the pelvic girdle and neck. Symptoms are usually symmetric. Inflammatory markers are mostly raised, and patients typically experience a swift response to glucocorticoids (1).

\section{IMAGING FINDINGS IN POLYMYALGIA RHEUMATICA}

In PMR, both joints and periarticular tissues can be involved. Arthritis is typically non-erosive and promptly responds to therapy (2), while on imaging the subacromialsubdeltoid (SAD) and trochanteric bursae appear often inflamed (1) (Figure 1). Despite the name of polymyalgia (pain in numerous muscles), the muscles have a normal appearance on imaging (3).

Routine radiographs of inflamed joints do not show abnormalities in patients with PMR (3). Scintigraphy has gone out of fashion with the advent of imaging techniques that provide better spatial resolution, such as ultrasonography (US) and magnetic res- onance imaging (MRI), but has high sensitivity in PMR patients not treated with glucocorticoids (4). ${ }^{18}$ F-Fluorodeoxyglucose positron emission tomography (PET) is not routinely used to image patients with PMR, but is indicated if large-vessel vasculitis is suspected. In addition, PET can also reveal inflammatory changes in articular and periarticular structures (3).

Imaging has substantially contributed to defining PMR as a disease mainly involving extra-articular structures. In fact, while mild synovitis is a known feature of PMR, it was noted that it can only partially explain the diffuse and severe discomfort of the proximal limb extremities experienced by PMR patients (5). A pivotal study by Salvarani et al. published back in 1997 clarified that the main source of pain and stiffness in PMR with shoulder symptoms was SAD bursitis (6). Using MRI, the authors investigated the shoulders of thirteen patients with PMR and of nine control patients with elderly onset rheumatoid arthritis as well as of ten age-matched unaffected controls. The main finding of this study was that SAD bursitis was present in $100 \%$ of PMR patients compared with only $22 \%$ of control patients, while the frequencies of joint synovitis and biceps tenosynovitis did not significantly differ between case and control patients. $\overline{\text { Corresponding author: }}$ Niccolò Possemato Rheumatology Unit, Arcispedale Santa Maria Nuova Viale Risorgimento, 80 42123 Reggio Emilia, Italy E-mail: niccolo.possemato@asmn.re.it 


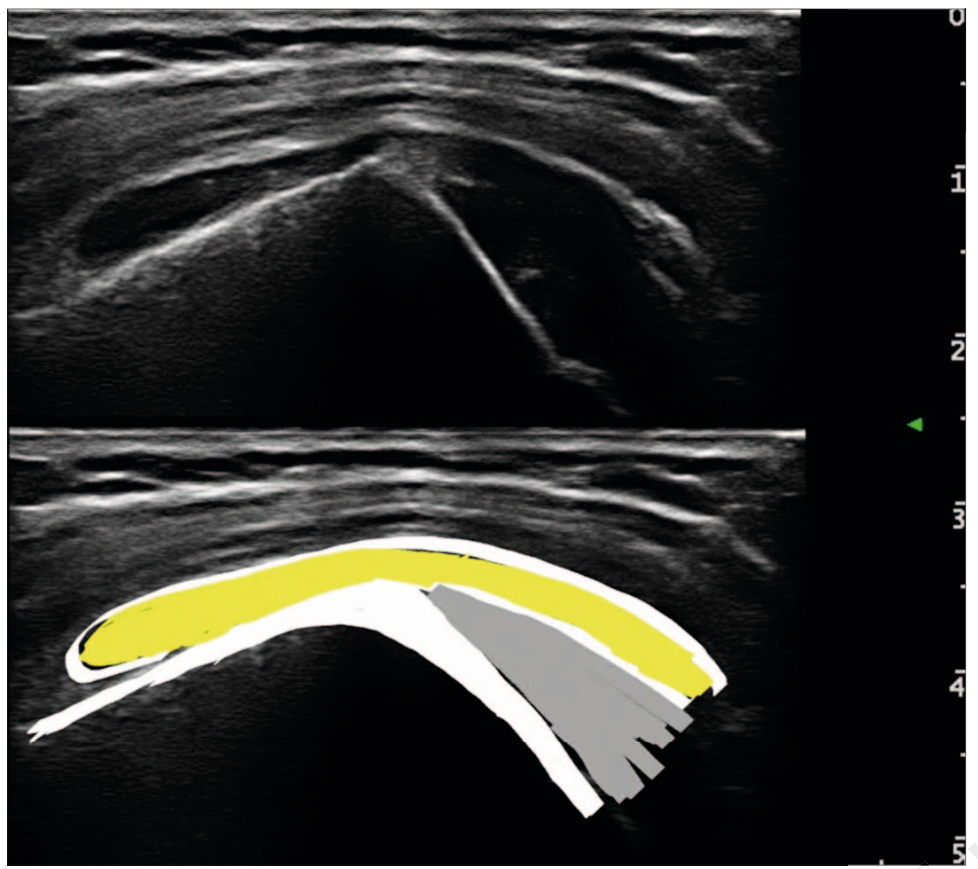

Figure 1 - Distension of the subacromial-deltoid bursa in a patient with PMR. In the second figure from top, the main anatomical structures are highlighted (the bursa in yellow, the supraspinatus tendon in grey and the bone and bursal margins in white).
Patients with PMR may also present with neck and, less commonly, with lower back pain, but the source of spinal pain has long not been recognized. Using PET, Blockmans et al. (7) had observed increased FGD accumulation at the level of the spinous processes in about one-half of PMR patients, but owing to the relatively low spatial resolution of PET the exact anatomical structures involved could not be identified. Two subsequent studies specifically looked at cervical (8) and lumbar (9) spine changes in patients with active PMR. Using MRI, Salvarani et al. were able to identify interspinous bursitis as the typical alteration of PMR patients. In the first study, twelve untreated PMR patients were compared with thirteen controls including patients with fibromyalgia, cervical osteoarthritis, ankylosing spondylitis and psoriatic spondylitis (8). In all patients with PMR, MRI showed the presence of fluid in the cervical interspinous bursae at C5-C7 level consistent with bursitis, while bursitis was noted in only $46 \%$ of control patients. Moderate to severe bursitis occurred significantly more frequently in PMR (83\%) than in control (31\%) patients. Similarly, MRI evidence of lumbar interspinous bursitis was detected in a subsequent study in $90 \%$ of 10 PMR patients, but in only $46 \%$ of a control group consisting of seven patients with spondyloarthropathy, two patients with spinal spondylosis and two patients with rheumatoid arthritis who had back pain (9). On the basis of these findings, it was proposed that PMR should be considered a disorder of extra-articular synovial structures $(5,10)$ and that joint synovitis might be secondary to shedding of proinflammatory molecules of extrasynovial membranes (10). From a nosological point of view, it was thus suggested that PMR might share a prevalent extra-articular synovial involvement with other conditions such as RS3PE (remitting seronegative symmetrical synovitis with pitting edema) and probably a subset of elderly onset rheumatoid arthritis, with important implications for the classification of arthritis in the elderly population (5).

Currently, US has gained a pivotal role in the assessment of PMR due to its capacity to visualize both joints and periarticular tissues, its low cost and widespread availability. Importantly, US in PMR has excellent reliability, with very high intra-observer (0.96) and inter-observer (0.99) intraclass correlation coefficients (11).

The most frequent US abnormalities are usually detected at shoulder level and are represented by SAD bursitis and long head of biceps tendon tenosynovitis (12). According to a recent review, SAD bursitis is found in about $70-100 \%$ of patients with PMR, while the prevalence of long head of biceps tendon tenosynovitis hovers around $45-100 \%$ (13). In this context, Rozin et al. described the two tram tracks sign, a specific US sign of active PMR. In some PMR patients, the two layers of the deltoid fascia and the two leaflets of the subdeltoid bursa, when viewed anteriorly with the shoulder extended, abducted and internally rotated, are seen as parallel hypoechogenic layers. This sign has been seen to resolve after treatment with glucocorticoids. Just how 
prevalent this sign is in PMR is as yet unknown (14).

At hip level, extra-articular involvement is the most relevant finding with the evidence of trochanteric bursitis that is present in a significantly higher number of cases with PMR than in controls (15). Other findings that have been reported are glenohumeral or hip joint effusions or tenosynovitis (15-18). Joint effusion can be also detected in the hips, knees, and wrists with prevalence rates of $40 \%, 38 \%$, and $18 \%$, respectively (19). Synovitis of hand and wrist are possible findings, but less helpful in differentiating PMR from other inflammatory conditions such as rheumatoid arthritis (RA). Joint involvement at feet level (metatarsophalangeal joints) is rare and should suggest another diagnosis.

MRI can also depict periarticular structures very well. In a study, MRI of the shoulders showed bilateral SAD bursitis in all PMR patients and biceps tenosynovitis in $67 \%$; glenohumeral synovitis was also detected in all patients (20). Similarly, at hip level using MRI, Cantini et al. demonstrated trochanteric bursitis in $100 \%$ and hip synovitis in $85 \%$ of PMR patients (15). US had the same sensitivity as MRI for the detection of trochanteric bursitis, whereas US findings of hip synovitis were present only in $45 \%$ of patients. A prevalent extra-articular pattern has also been demonstrated in the hands of patients with PMR using MRI. Cimmino et al. reported tenosynovitis in $67 \%$ of 15 PMR patients versus $30 \%$ of unaffected controls; extensor tenosynovitis was seen in 53\% and flexor tenosynovitis in $60 \%$ of PMR patients, although only the rate of extensor tenosynovitis was significantly higher than in controls (21). In contrast, signs of synovitis were comparable in PMR patients and controls. Likewise, in patients with florid, PMR-associated RS3PE (remitting seronegative symmetrical synovitis with pitting edema), the most common MRI findings are extensor tenosynovitis, followed in decreasing order by hand joint arthritis and flexor tenosynovitis (22).

A periarticular pattern of PMR has recently been highlighted in a study by Mackie et al. (23). In this study, 22 patients with PMR and 16 with RA underwent whole-body, multiple-joint, 3T MRI. The authors identified a pattern of symmetrical extracapsular inflammation at the level of the greater trochanter, acetabulum, ischial tuberosity and/or symphysis pubis in 64\% of PMR. This pattern was associated with higher pre-treatment interleukin- 6 and C-reactive protein levels and a complete response to glucocorticoids, as well as with better posttreatment fatigue and function. However, patients presenting with such a pattern were more likely to require glucocorticoid treatment for $>1$ year.

PET is also a valuable tool in identifying inflammatory changes in PMR as well as in disclosing subclinical large vessel vasculitis (Figure 2). In PMR, the increased FDG uptake is mainly related to bursitis (peri-

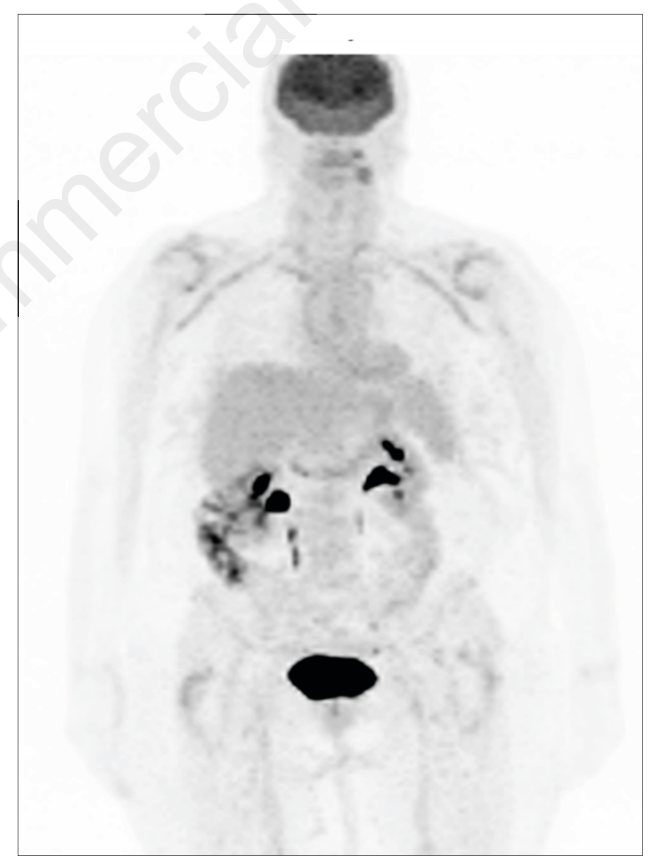

Figure 2 - PET/CT image in a patient with PMR associated vasculitis. The FDG uptake pattern at the level of the periarticular structures of the shoulders, hips (bursitis) and humeral head (synovitis) is consistent with PMR. In this patient, PET/CT also shows an associated large vessel vasculitis (increased vascular FDG uptake at the thoracic aorta, subclavian arteries and axillary arteries, score 2 according to Meller $\mathrm{J}$ et al. Eur $\mathrm{J}$ Nucl Med Mol Imaging, 2003). 
articular accumulation pattern) (24). In one of the first papers based on PET published by Blockmans et al., high 18F-FDG uptake was detected in shoulders in 33/35 (94\%) patients and in hips in 31/35 (89\%) patients as well as in the spinous process of the vertebrae in 51\% (7). PET can allow assessment of deep bursae, not easily visible with US, such as ischial tuberosities, lumbar spinous processes and greater trochanters. Positive results at this level seems to be highly sensitive $(85.7 \%)$ and specific (88.2\%) for the diagnosis of PMR, according to Yamashita et al. (25).

Rehak et al. recently described various patterns of PET findings in 67 patients who fulfilled Healey's criteria for PMR (26). The authors found articular involvement in proximal joints in $88.1 \%$ of patients (86.6\% shoulders, $70.1 \%$ hips and $46.3 \%$ sternoclavicular joints). Vascular involvement was seen in $40.3 \%$ of patients. Extra-articular involvement was present in ischiogluteal bursae in $52.2 \%$ of patients, around the symphysis and ventral to pubic bones in $7.5 \%$ of patients, in spinous interspaces of cervical vertebrae in $19.4 \%$ of patients and in lumbar vertebrae in $56.7 \%$ of patients.

Imaging studies are particularly useful in securing diagnosis of PMR when the inflammatory markers are normal. Cantini et al. demonstrated that MRI and US in PMR patients with normal or high erythrocyte sedimentation rate reveal similar inflammatory shoulder lesions (27).

PET is especially helpful in disclosing vascular involvement associated with PMR, since it can visualize almost all arteries including the deeper vessels. Blockmans et al. showed that about onethird of 35 patients with isolated PMR had increased vascular FDG uptake (predominantly in the subclavian arteries) (28). However, uptake intensity was less marked than in giant cell arteritis, with only $2 / 35(6 \%)$ patients showing intense vascular uptake. Moosig et al. (29) described increased FDG vascular uptake in 12 out of 13 PMR patients in the aorta and its major branches, but in another study, only $2 / 14(14 \%)$ untreated patients with
PMR had increased FDG vascular uptake (25). Importantly, PMR patients without clinical evidence of arteritis but with imaging signs of giant cell arteritis do not appear prone to developing vascular ischemic complications.

\section{ROLE OF IMAGING IN THE CLASSIFICATION CRITERIA OF POLYMYALGIA RHEUMATICA}

A gold standard diagnostic test for PMR is lacking. Moreover, PMR can be mimicked by numerous other conditions, many of which may also respond in some degree to glucocorticoids. Various classification criteria have been proposed for diagnosing PMR, including those by Chuang (30), Healey (31) and Bird (32). However, none of these criteria have been validated or been widely accepted. Therefore, new classification criteria have been developed under the aegis of the American College of Rheumatology and of European League Against Rheumatism (33).

In patients aged 50 years or older presenting with bilateral shoulder aching and raised inflammatory markers, these criteria had $68 \%$ sensitivity and $78 \%$ specificity for PMR. US was not mandatory, but when ultrasonography findings consistent with PMR were included, sensitivity remained virtually unchanged at $66 \%$, whereas specificity increased to $81 \%$. US findings deemed consistent with PMR included at least one shoulder with subdeltoid bursitis and/or biceps tenosynovitis and/or glenohumeral synovitis (either posterior or axillary) and at least one hip with synovitis and/or trochanteric bursitis. Macchioni et al. recently demonstrated the increased specificity of the clinical criteria if integrated with ultrasonography (34). Patients with PMR were compared to those with other joint inflammatory disorders including rheumatoid arthritis (RA). Adding ultrasonography, specificity increased from $81.5 \%$ to $91.3 \%$ in total cases and from $79.7 \%$ to $89.9 \%$ in RA. These findings suggest that ultrasonography examination is helpful in increasing the specificity of a clinical diagnosis of PMR. 


\section{ROLE OF IMAGING IN THE DIFFERENTIAL DIAGNOSIS OF POLYMYALGIA RHEUMATICA}

Current published data suggest a possible role for imaging in differentiating PMR from other rheumatic conditions that may present with a polymyalgic onset. Falsetti et al. in 2011 demonstrated an improvement of diagnostic sensitivity for PMR when US assessment was used. The authors suggested a predictive model of US evaluation to classify PMR patients, including the presence of SAD bursitis, low frequency of wrist, metacarpophalangeal and metatarsophalangeal effusion/synovitis, low frequency of Achilles enthesitis, low frequency of knee menisci chondrocalcinosis, and tendinous calcaneal calcifications, and low hypervascularization at power-Doppler US analysis in the wrist $(13,18)$.

In another study, Falsetti et al. compared US findings in patients with PMR versus those with RA or spondyloarthropathies (SpA) (35). They found that enthesitis was more frequent in $\mathrm{SpA}$, while synovitis of the elbow, knee, and wrist was significantly more common in patients with RA and SpA patients compared with those with PMR. At shoulder level, a study that compared PMR with RA and psoriatic arthritis (PsA) patients found that bilateral SAD bursitis was universal $(100 \%)$ in patients with PMR, whereas only $20 \%$ of patients of RA patients and no PsA patients had such findings (20). In contrast, no significant difference was detected in the distribution of biceps tenosynovitis among the study groups. Another study that looked at shoulder US findings in patients with PMR and RA confirmed that SAD bursitis was more specific to PMR (36). In this study, bilateral SAD bursitis was revealed in $37 \%$ of PMR patients, but only in $3 \%$ of patients with RA, while bilateral biceps tenosynovitis was present in $30 \%$ of PMR patients versus none of the RA controls. A semiquantitative scoring system for evaluating periarticular shoulder US inflammatory signs has recently been proposed by Suzuki et al. (37). Using a four-point scale scoring the hyperemia of the suprascapu- laris tendon, the authors demonstrated that inflammation in PMR was predominantly localized to the periarticular tissue compared with elderly-onset RA. The scoring showed good intra- and inter-observer reliability.

MRI is also an excellent imaging technique for PMR, due to its capacity to visualize inflamed tissues at joint and bursae level, and has proved useful for the purpose of differential diagnosis. In 2001, McGonagle et al. published an MRI study comparing the anatomical sites of inflammatory changes in the shoulders of 14 patients with early PMR and 14 with RA using fat suppression MRI (38). The authors described a common involvement of synovial joints in PMR and RA and a slightly higher frequency of bursitis in PMR patients. Interestingly, they also described the presence of inflammatory changes in the soft tissues, defined as extracapsular changes.

In 2007, the same group published an interesting paper evaluating the relationship between synovial and extracapsular inflammation in PMR and early RA using contrast-enhanced and fat suppression MRI (39). The results confirmed a much greater degree of gadolinium enhancement in the extracapsular tissues in the PMR patients. A surprising finding was that MRIdetermined erosion and bone edema were equally common in both groups, while it has been recognized that PMR synovitis is usually non-erosive at standard X-ray evaluation (22). This finding may be related to the capacity of MRI to detect even small erosions, which can be found even in normal subjects (21).

Approximately $16-21 \%$ of patients with PMR have giant cell arteritis (1). Imaging methods can aid in detecting those PMR patients that have an associated vasculitis. US can be a valuable tool for a correct diagnosis of these patients, especially when superficial arteries are involved, with a sensitivity ranging from $55 \%$ to $100 \%$ and specificity ranging from $78 \%$ to $100 \%$ (40). In large vessel vasculitis, the inflammatory infiltrate of the vessel wall results in the loss of the normal echostructure of the intima-media complex. The major so- 


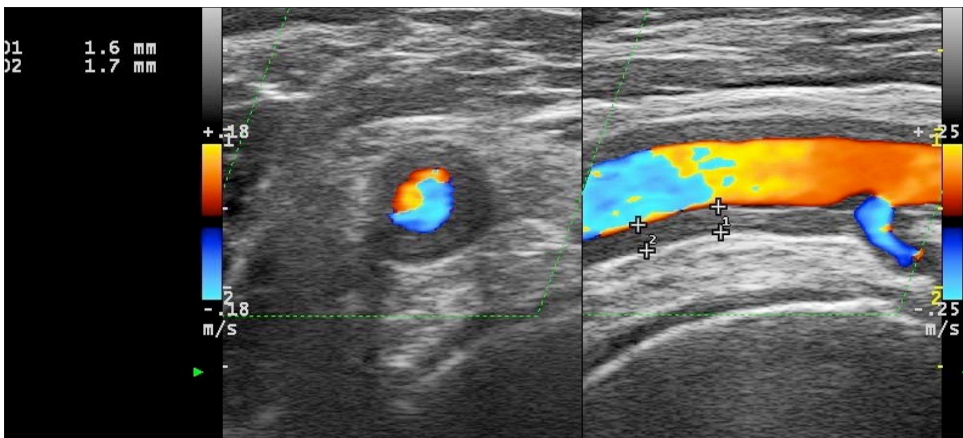

Figure 3 - Vasculitis of the axillary arteries in a patient with PMR. Note both in the transversal (left) and in the longitudinal view (right) the concentric thickening of the intima-media complex of the axillary artery at the humeral head level.

nographic signs are the thickening of the arterial wall with disappearance of the trilaminar structure of the intima-media complex, the presence of a perivascular halo, and the presence of stenoses or vascular dilatations (Figure 3) $(41,42)$.

PET, too, can be used to detect an underlying large-vessel vasculitis as well as to depict the pattern of involvement of periarticular structures in PMR. In terms of differential diagnosis, PET has been suggested as useful in differentiating PMR from elderly onset RA, with important implications in terms of prognosis and treatment. Yamashita et al. compared FDG accumulation sites at PET between 27 patients with PMR and 10 with elderly onset RA. In PMR, abnormal accumulation at the ischial tuberosity, vertebral spinous processes, and iliopectineal bursa was significantly higher compared with RA, while shoulder involvement had a similar prevalence (25). Similarly, Wakura et al. suggested that an abnormal FDG accumulation at the entheses of the girdle such as enthesis of the pectineus muscle, and enthesis of the rectus femoris muscle might assist in differentiating PMR from EORA (43).

\section{ROLE OF IMAGING IN ASSESSING TREATMENT RESPONSE IN POLYMYALGIA RHEUMATICA}

There is limited evidence on the role of imaging in monitoring patients with PMR.
In a prospective study containing 53 patients evaluated clinically, serologically and by US before and after (at 4 weeks and at 12 weeks) glucocorticoid therapy, clinical, laboratory and ultrasonography decreased in parallel over time (11). US inflammatory findings showed similar or better sensitivity to change than clinical and laboratory markers of PMR activity (11). However, in another prospective study on 57 newly diagnosed patients with PMR, while glucocorticoids significantly reduced the frequency and the severity of SAD bursitis, long head biceps tenosynovitis, and glenohumeral synovitis, a sizeable $59 \%$ of 44 patients in clinical remission or in low disease activity had persistent inflammatory lesions at followup US (24). No association was found between the persistence of US inflammatory changes and the risk of relapses or recurrences, although a positive power-Doppler signal at diagnosis was significantly associated with the subsequent occurrence of relapses or recurrences. Similarly, FDG uptake at PET (in the shoulders, hips, and spinous processes) was shown to decrease following the institution of glucocorticoids therapy; however, basal FDG uptake did not predict the risk of relapses over time (28).

Recently, Palard-Novello et al. suggested a role for PET in assessing the efficacy of tocilizumab in the treatment of PMR (44). Patients enrolled in a multicentric clinical trial underwent PET at baseline, after the first infusion of tocilizumab (week 2) and after the last infusion (week 12). Eighteen patients could be evaluated. At week 0 , high FDG uptake was found at the shoulder (89\% of patients) and pelvic ( $94 \%$ of patients) girdle as well as in the cervical spinous processes (56\% of patients). After the first infusion, SUV max significantly decreased and decreased further by week 12 , although in a lesion-based analysis no significant decrease was observed in the shoulders and cervical spinous processes. Further studies are required to fully elucidate the role of imaging in gauging response to treatment and monitoring PMR patients over time. 


\section{FUTURE PROSPECTS}

Imaging appears to increase the specificity of a clinical diagnosis of PMR, and to discriminate better between PMR and its mimickers at an early stage. Therefore, imaging can conceivably play an important role in selecting PMR patients for clinical trials with greater accuracy. As suggested by Buttgereit et al. (45), imaging including MRI could help to identify a fairly homogeneous subset of patients with PMR with potential value for defining eligibility for early clinical trials of targeted therapies. In addition, imaging could also complement clinical and laboratory assessments of patients over time, including response to therapy.

\section{REFERENCES}

1. Salvarani C, Cantini F, Hunder GG. Polymyalgia rheumatica and giant-cell arteritis. Lancet. 2008; 372: 234-45.

2. Salvarani C, Cantini F, Macchioni P, et al. Distal musculoskeletal manifestations in polymyalgia rheumatica: a prospective followup study. Arthritis Rheum. 1998; 41: 1221-6.

3. Camellino D, Cimmino MA. Imaging of polymyalgia rheumatica: indications on its pathogenesis, diagnosis and prognosis. Rheumatology (Oxford). 2012; 51: 77-86.

4. O'Duffy JD, Wahner HW, Hunder GG. Joint imaging in polymyalgia rheumatica. Mayo Clin Proc. 1976; 51: 519-24.

5. Salvarani C, Cantini F, Olivieri I, Hunder GS. Polymyalgia rheumatica: a disorder of extraarticular synovial structures? J Rheumatol. 1999; 26: 517-21.

6. Salvarani C, Cantini F, Olivieri I, et al. Proximal bursitis in active polymyalgia rheumatica. Ann Intern Med. 1997; 127: 27-31.

7. Blockmans D, De Ceuninck L, Vanderschueren S, et al. Repetitive 18-fluorodeoxyglucose positron emission tomography in isolated polymyalgia rheumatica: a prospective study in 35 patients. Rheumatology (Oxford). 2007; 46: 672-7.

8. Salvarani C, Barozzi L, Cantini F, et al. Cervical interspinous bursitis in active polymyalgia rheumatica. Ann Rheum Dis. 2008; 67: 758-61.

9. Salvarani C, Barozzi L, Boiardi L, et al. Lumbar interspinous bursitis in active polymyalgia rheumatica. Clin Exp Rheumatol. 2013; 31: 526-31.

10. Salvarani C, Olivieri I, Cantini F, Hunder GG. Classification of inflammatory arthritis. Lancet. $1998 ; 352$ : 1938.

11. Jimenez-Palop M, Naredo E, Humbrado L, et al. Ultrasonographic monitoring of response to therapy in polymyalgia rheumatica. Ann Rheum Dis. 2010; 69: 879-82.

12. Ruta S, Rosa J, Navarta DA, et al. Ultrasound assessment of new onset bilateral painful shoulder in patients with polymyalgia rheumatica and rheumatoid arthritis. Clin Rheumatol. 2012; 31: 1383-7.

13. Iagnocco A, Finucci A, Ceccarelli F, et al. Musculoskeletal ultrasound in the evaluation of polymyalgia rheumatica. Med Ultrason. 2015; 17: 361-6.

14. Rozin AP. US imaging of shoulder fasciitis due to polymyalgia rheumatica. Neth J Med. 2008; 66: 88.

15. Cantini F, Niccoli L, Nannini C, et al. Inflammatory changes of hip synovial structures in polymyalgia rheumatica. Clin Exp Rheumatol. $2005 ; 23$ : 462-8.

16. Frediani B, Falsetti P, Storri L, et al. Evidence for synovitis in active polymyalgia rheumatica: sonographic study in a large series of patients. J Rheumatol. 2002; 29: 123-30.

17. Cantini F, Salvarani C, Olivieri I, et al. Shoulder ultrasonography in the diagnosis of polymyalgia rheumatica: a case-control study. J Rheumatol. 2001; 28: 1049-55.

18. Falsetti P, Acciai C, Volpe A, Lenzi L. Ultrasonography in early assessment of elderly patients with polymyalgic symptoms: a role in predicting diagnostic outcome? Scand J Rheumatol. 2011; 40: 57-63.

19. Delle SA, Riente L, Filippucci E, et al. Ultrasound imaging for the rheumatologist. XV. Ultrasound imaging in vasculitis. Clin Exp Rheumatol. 2008; 26: 391-4.

20. Cantini F, Salvarani C, Niccoli L, et al. Fat suppression magnetic resonance imaging in shoulders of patients with polymyalgia rheumatica. J Rheumatol. 2004; 31: 120-4.

21. Cimmino MA, Parodi M, Zampogna G, et al. Polymyalgia rheumatica is associated with extensor tendon tenosynovitis but not with synovitis of the hands: a magnetic resonance imaging study. Rheumatology (Oxford). 2011; 50: 494-9.

22. Cantini F, Salvarani C, Olivieri I, et al. Remitting seronegative symmetrical synovitis with pitting oedema (RS3PE) syndrome: a prospective follow up and magnetic resonance imaging study. Ann Rheum Dis. 1999; 58: 230-6.

23. Mackie SL, Pease CT, Fukuba E, et al. Wholebody MRI of patients with polymyalgia rheumatica identifies a distinct subset with complete patient-reported response to glucocorticoids. Ann Rheum Dis. 2015; 74: 2188-92.

24. Macchioni P, Catanoso MG, Pipitone N, et al. Longitudinal examination with shoulder ultrasound of patients with polymyalgia rheumatica. Rheumatology (Oxford). 2009; 48: 1566-9. 
25. Yamashita H, Kubota K, Takahashi Y, et al. Whole-body fluorodeoxyglucose positron emission tomography/computed tomography in patients with active polymyalgia rheumatica: evidence for distinctive bursitis and largevessel vasculitis. Mod Rheumatol. 2012; 22: 705-11.

26. Rehak Z, Vasina J, Nemec P, et al. Various forms of ${ }^{18} \mathrm{~F}-\mathrm{FDG}$ PET and PET/CT findings in patients with polymyalgia rheumatica. Biomed Pap Med Fac Univ Palacky Olomouc Czech Repub. 2015; 159: 629-36.

27. Cantini F, Salvarani C, Olivieri I, et al. Inflamed shoulder structures in polymyalgia rheumatica with normal erythrocyte sedimentation rate. Arthritis Rheum. 2001; 44: 1155-9.

28. Blockmans D, De Ceuninck L, Vanderschueren S, et al. Repetitive 18F-fluorodeoxyglucose positron emission tomography in giant cell arteritis: a prospective study of 35 patients. Arthritis Rheum. 2006; 55: 131-7.

29. Moosig F, Czech N, Mehl C, et al. Correlation between 18-fluorodeoxyglucose accumulation in large vessels and serological markers of inflammation in polymyalgia rheumatica: a quantitative PET study. Ann Rheum Dis. 2004; 63: 870-3.

30. Chuang TY, Hunder GG, Ilstrup DM, Kurland LT. Polymyalgia rheumatica: a 10-year epidemiologic and clinical study. Ann Intern Med. 1982; 97: 672-80.

31. Healey LA. Long-term follow-up of polymyalgia rheumatica: evidence for synovitis. Semin Arthritis Rheum. 1984; 13: 322-8.

32. Bird HA, Esselinckx W, Dixon AS, et al. An evaluation of criteria for polymyalgia rheumatica. Ann Rheum Dis. 1979; 38: 434-9.

33. Dasgupta B, Cimmino MA, Maradit-Kremers $\mathrm{H}$, et al. 2012 provisional classification criteria for polymyalgia rheumatica: a European League Against Rheumatism/American College of Rheumatology collaborative initiative. Ann Rheum Dis. 2012; 71: 484-92.

34. Macchioni P, Boiardi L, Catanoso M, et al. Performance of the new 2012 EULAR/ACR classification criteria for polymyalgia rheumatica: comparison with the previous criteria in a single-centre study. Ann Rheum Dis. 2014; 73: 1190-3.

35. von Knorring J. Treatment and prognosis in polymyalgia rheumatica and temporal arteri- tis. A ten-year survey of 53 patients. Acta Med Scand. 1979; 205: 429-35.

36. Ruta S, Rosa J, Navarta DA, et al. Ultrasound assessment of new onset bilateral painful shoulder in patients with polymyalgia rheumatica and rheumatoid arthritis. Clin Rheumatol. 2012; 31: 1383-7.

37. Suzuki T, Yoshida R, Okamoto A, Seri Y. Semiquantitative evaluation of extrasynovial soft tissue inflammation in the shoulders of patients with polymyalgia rheumatica and elderly-onset rheumatoid arthritis by power doppler ultrasound. Biomed Res Int. 2017; 2017: 4272560.

38. McGonagle D, Pease C, Marzo-Ortega H, et al. Comparison of extracapsular changes by magnetic resonance imaging in patients with rheumatoid arthritis and polymyalgia rheumatica. J Rheumatol. 2001; 28: 1837-41.

39. Marzo-Ortega H, Rhodes LA, Tan AL, et al. Evidence for a different anatomic basis for joint disease localization in polymyalgia rheumatica in comparison with rheumatoid arthritis. Arthritis Rheum 2007; 56: 3496-501.

40. Buttgereit F, Dejaco C, Matteson EL, Dasgupta B. Polymyalgia rheumatica and giant cell arteritis: a systematic review. JAMA. 2016; 315: 2442-58.

41. Pipitone N, Versari A, Salvarani C. Role of imaging studies in the diagnosis and follow-up of large-vessel vasculitis: an update. Rheumatology (Oxford). 2008; 47: 403-8.

42. Germano G, Monti S, Ponte C, et al. The role of ultrasound in the diagnosis and follow-up of large-vessel vasculitis: an update. Clin Exp Rheumatol. 2017; 35: 194-8.

43. Wakura D, Kotani T, Takeuchi T, et al. Differentiation between polymyalgia rheumatica (PMR) and elderly-onset rheumatoid arthritis using 18f-fluorodeoxyglucose positron emission tomography/computed tomography: Is enthesitis a new pathological lesion in PMR? PLoS One. 2016; 11: e0158509.

44. Palard-Novello X, Querellou S, Gouillou M, et al. Value of ${ }^{18}$ F-FDG PET/CT for therapeutic assessment of patients with polymyalgia rheumatica receiving tocilizumab as first-line treatment. Eur J Nucl Med Mol Imaging. 2016; 43: 773-9.

45. Buttgereit F, Matteson EL. Imaging: Wholebody MRI undresses polymyalgia rheumatica. Nat Rev Rheumatol. 2016; 12: 140-1. 